\title{
Observational Constraints on Teleparallel Dark Energy
}

\author{
Chao-Qiang Geng ${ }^{a, b, c}$ Chung-Chi Lee ${ }^{a, c}$ Emmanuel N. Saridakis ${ }^{d, b}$ \\ ${ }^{a}$ Department of Physics, National Tsing Hua University, Hsinchu, Taiwan 300 \\ ${ }^{b}$ National Center for Theoretical Sciences, Hsinchu, Taiwan 300 \\ ${ }^{c}$ Kavli Institute for Theoretical Physics China, Chinese Academy of Science, Beijing 100190 \\ ${ }^{d}$ CASPER, Physics Department, Baylor University, Waco, TX 76798-7310, USA \\ E-mail: geng@phys.nthu.edu.tw, g9522545@oz.nthu.edu.tw, \\ Emmanuel_Saridakis@baylor.edu
}

Abstract: We use data from Type Ia Supernovae (SNIa), Baryon Acoustic Oscillations (BAO), and Cosmic Microwave Background (CMB) observations to constrain the recently proposed teleparallel dark energy scenario based on the teleparallel equivalent of General Relativity, in which one adds a canonical scalar field, allowing also for a nonminimal coupling with gravity. Using the power-law, the exponential and the inverse hyperbolic cosine potential ansatzes, we show that the scenario is compatible with observations. In particular, the data favor a nonminimal coupling, and although the scalar field is canonical the model can describe both the quintessence and phantom regimes.

KEYwords: Modified gravity, dark energy, phantom-divide crossing, $\mathrm{f}(\mathrm{T})$ gravity, nonminimal coupling 


\section{Contents}

1 Introduction. $\quad 1$

2 Teleparallel Dark Energy $\quad 2$

3 Observational Constraints 4

4 Conclusions $\quad 9$

A Analysis method for the observational data 10

\section{Introduction.}

Recent cosmological observations [1-5] indicate that the observable universe experiences an accelerated expansion. Although the simplest way is the consideration of a cosmological constant, there are two alternative ways to explain this behavior. The first is to modify the content of the universe by introducing the dark energy sector, which can be based on a canonical scalar field (quintessence) [6-22], a phantom field [23-28], or on the combination of both these fields in a unified scenario called quintom [29-35] (see [36] for a review). The other approach is to modify the gravitational sector itself (see [37] for a review and references therein).

The teleparallel dark energy is a recently proposed scenario that tries to incorporate the dark energy sector [38]. It is based on the "teleparallel" equivalent of General Relativity (TEGR), that is on its torsion instead of curvature formulation [39, 40], in which one adds a canonical scalar field, and the dark energy sector is attributed to this field. In the case where the field is minimally coupled to gravity the scenario is completely equivalent to the standard quintessence [6-11], both at the background and perturbation levels. However, in the case where one switches on the nonminimal coupling between the field and the torsion scalar, that is the only suitable gravitational scalar in TEGR, the resulting scenario has a richer structure, exhibiting quintessence-like or phantom-like behavior, or experiencing the phantom-divide crossing [38]. The richer structure is manifested in the absence of a conformal transformation to an equivalent minimally-coupled model with transformed field and potential, which is known to be able to describe only the quintessence regime.

Since the teleparallel dark energy exhibits interesting cosmological behavior, in the present work we use observations in order to constrain the parameters of the model. In particular, we use data from Type Ia Supernovae (SNIa), Baryon Acoustic Oscillations (BAO), and Cosmic Microwave Background (CMB) to plot likelihood-contours for the present dark-energy equation-of-state, matter density parameter and nonminimal coupling parameters, respectively. 
The paper is organized as follows: In Section 2 we present the scenario of the teleparallel dark energy and derive the relevant equations for the cosmological evolution. In Section 3 we use observational data to produce likelihood-contours of the model parameters. Finally, Section 4 is devoted to the summary of our results.

\section{Teleparallel Dark Energy}

Let us briefly review the teleparallel dark energy. As we stated in Introduction, it is based on the "teleparallel" equivalent of General Relativity (TEGR) [39, 40], in which instead of using the torsionless Levi-Civita connection one uses the curvatureless Weitzenböck one. The dynamical objects are the four linearly independent vierbeins (these are parallel vector fields, referred to as the appellations "teleparallel" or "absolute parallelism"). It is interesting to note that the torsion tensor is formed solely from products of the first derivatives of the tetrad.

In particular, the vierbein field $\mathbf{e}_{A}\left(x^{\mu}\right)$ forms an orthonormal basis for the tangent space at each point $x^{\mu}$, that is $\mathbf{e}_{A} \cdot \mathbf{e}_{B}=\eta_{A B}$, where $\eta_{A B}=\operatorname{diag}(1,-1,-1,-1)$, and furthermore the vector $\mathbf{e}_{A}$ can be analyzed with the use of its components $e_{A}^{\mu}$ in a coordinate basis, that is $\mathbf{e}_{A}=e_{A}^{\mu} \partial_{\mu}{ }^{1}$. In such a construction, the metric tensor is obtained from the dual vierbein as

$$
g_{\mu \nu}(x)=\eta_{A B} e_{\mu}^{A}(x) e_{\nu}^{B}(x)
$$

Consequently, the torsion tensor of the Weitzenböck connection $\stackrel{\mathrm{w}}{\Gamma}_{\nu \mu}^{\lambda}$ [43] reads

$$
T_{\mu \nu}^{\lambda}=\stackrel{\mathrm{w}}{\Gamma}_{\nu \mu}^{\lambda}-\stackrel{\mathrm{w}}{\Gamma}_{\mu \nu}^{\lambda}=e_{A}^{\lambda}\left(\partial_{\mu} e_{\nu}^{A}-\partial_{\nu} e_{\mu}^{A}\right),
$$

where $\stackrel{\mathrm{w}}{\Gamma}_{\nu \mu}^{\lambda} \equiv e_{A}^{\lambda} \partial_{\mu} e_{\nu}^{A}$.

In the present formalism, all the information concerning the gravitational field is included in the torsion tensor $T_{\mu \nu}^{\lambda}$. As described in [40], the "teleparallel Lagrangian" can be constructed from this torsion tensor under the assumptions of invariance under general coordinate transformations, global Lorentz transformations, and the parity operation, along with requiring the Lagrangian density to be the second order in the torsion tensor. In particular, it is the torsion scalar $T$, given by [39, 40, 44, 45]:

$$
\mathcal{L}=T=\frac{1}{4} T^{\rho \mu \nu} T_{\rho \mu \nu}+\frac{1}{2} T^{\rho \mu \nu} T_{\nu \mu \rho}-T_{\rho \mu}{ }^{\rho} T_{\nu \mu}^{\nu} .
$$

The simplest action in a universe governed by teleparallel gravity is

$$
S=\int d^{4} x e\left[\frac{T}{2 \kappa^{2}}+\mathcal{L}_{m}\right]
$$

where $e=\operatorname{det}\left(e_{\mu}^{A}\right)=\sqrt{-g}$ (one could also include a cosmological constant). Variation with respect to the vierbein fields provides equations of motion, which are exactly the same as

\footnotetext{
${ }^{1}$ We follow the notation of $[41,42]$, that is Greek indices $\mu, \nu, \ldots$ and capital Latin indices $A, B, \ldots$ run over all coordinate and tangent space-time $0,1,2,3$, while lower case Latin indices (from the middle of the alphabet) $i, j, \ldots$ and lower case Latin indices (from the beginning of the alphabet) $a, b, \ldots$ run over spatial and tangent space coordinates $1,2,3$, respectively. Finally, we use the metric signature $(+,-,-,-)$.
} 
those of GR for every geometry choice, and that is why the theory is called "teleparallel equivalent to General Relativity".

In principle one has two ways of generalizing the action (2.4), inspired by the corresponding procedures of the standard General Relativity. The first is to replace $T$ by an arbitrary function $f(T)$ [41, 42, 46-72], similar to $f(R)$ extensions of GR, and obtain new interesting terms in the field equations. The other, on which we focus in the present work, is to add a canonical scalar field in (2.4), similar to the GR quintessence, allowing for a nonminimal coupling between it and gravity. This field will constitute the dark energy sector, and thus the corresponding scenario is called "teleparallel dark energy" [38]. In particular, the action will simply read:

$$
S=\int \mathrm{d}^{4} x e\left[\frac{T}{2 \kappa^{2}}+\frac{1}{2}\left(\partial_{\mu} \phi \partial^{\mu} \phi+\xi T \phi^{2}\right)-V(\phi)+\mathcal{L}_{m}\right] .
$$

Concerning the nonminimal coupling we emphasize that the nonminimal coupling will be between the torsion and the scalar field (similar to the standard nonminimal quintessence where the scalar field couples to the Ricci scalar).

Variation of the action (2.5) with respect to the vierbein fields yields the equations of motion

$$
\begin{gathered}
\left(\frac{2}{\kappa^{2}}+2 \xi \phi^{2}\right)\left[e^{-1} \partial_{\mu}\left(e e_{A}^{\rho} S_{\rho}^{\mu \nu}\right)-e_{A}^{\lambda} T^{\rho}{ }_{\mu \lambda} S_{\rho}{ }^{\nu \mu}-\frac{1}{4} e_{A}^{\nu} T\right] \\
-e_{A}^{\nu}\left[\frac{1}{2} \partial_{\mu} \phi \partial^{\mu} \phi-V(\phi)\right]+e_{A}^{\mu} \partial^{\nu} \phi \partial_{\mu} \phi \\
+4 \xi e_{A}^{\rho} S_{\rho}^{\mu \nu} \phi\left(\partial_{\mu} \phi\right)=e_{A}^{\rho} \stackrel{\mathrm{em}}{T}_{\rho}^{\nu}
\end{gathered}
$$

where $\stackrel{\mathrm{em}}{T}_{\rho}^{\nu}$ stands for the usual energy-momentum tensor. Therefore, for a flat FriedmannRobertson-Walker (FRW) background metric

$$
d s^{2}=d t^{2}-a^{2}(t) \delta_{i j} d x^{i} d x^{j}
$$

and a vierbein choice of the form

$$
e_{\mu}^{A}=\operatorname{diag}(1, a, a, a)
$$

where $t$ is the cosmic time, $x^{i}$ are the comoving spatial coordinates and $a(t)$ is the scale factor, we obtain the corresponding Friedmann equations:

$$
\begin{aligned}
& H^{2}=\frac{\kappa^{2}}{3}\left(\rho_{\phi}+\rho_{m}\right), \\
& \dot{H}=-\frac{\kappa^{2}}{2}\left(\rho_{\phi}+p_{\phi}+\rho_{m}+p_{m}\right),
\end{aligned}
$$

where $H=\dot{a} / a$ is the Hubble parameter and a dot denotes differentiation with respect to $t$. In these expressions, $\rho_{m}$ and $p_{m}$ are the matter energy density and pressure, respectively, following the standard evolution equation $\dot{\rho}_{m}+3 H\left(1+w_{m}\right) \rho_{m}=0$, with $w_{m}=p_{m} / \rho_{m}$ the 
matter equation-of-state parameter. Additionally, we have introduced the energy density and pressure of the scalar field

$$
\begin{aligned}
\rho_{\phi} & =\frac{1}{2} \dot{\phi}^{2}+V(\phi)-3 \xi H^{2} \phi^{2}, \\
p_{\phi} & =\frac{1}{2} \dot{\phi}^{2}-V(\phi)+4 \xi H \phi \dot{\phi}+\xi\left(3 H^{2}+2 \dot{H}\right) \phi^{2} .
\end{aligned}
$$

Moreover, variation of the action with respect to the scalar field provides its evolution equation, namely:

$$
\ddot{\phi}+3 H \dot{\phi}+6 \xi H^{2} \phi+V^{\prime}(\phi)=0 .
$$

Note that in the above expressions we have used the useful relation $T=-6 H^{2}$, which straightforwardly arises from the calculation of (2.3) for the flat FRW geometry.

In this scenario, similar to the standard quintessence, dark energy is attributed to the scalar field, and thus its equation-of-state parameter $\left(w_{D E}\right)$ reads:

$$
w_{D E} \equiv w_{\phi}=\frac{p_{\phi}}{\rho_{\phi}} .
$$

As a result, one can see that the scalar field evolution (2.13) leads to the standard relation

$$
\dot{\rho}_{\phi}+3 H\left(1+w_{\phi}\right) \rho_{\phi}=0 .
$$

The teleparallel dark energy proves to exhibit a very interesting cosmological implication $[38,73]$. In the minimally-coupled case the cosmological equations coincide with those of the standard quintessence, both at the background and perturbation levels. However, when the nonminimal coupling is switched on, one can obtain a dark energy sector being quintessence-like, phantom-like, or experiencing the phantom-divide crossing during evolution, a behavior that is much richer comparing to General Relativity (GR) with a scalar field [38]. Therefore, it is both interesting and necessary to use observations to constrain the parameters of the scenario. This is performed in the next section.

\section{Observational Constraints}

We use Type Ia Supernovae (SNIa) from the Supernova Cosmology Project (SCP) Union2 compilation [74], Baryon Acoustic Oscillations (BAO) data from the Two-Degree Field Galaxy Redshift Survey (2dFGRS) and the Sloan Digital Sky Survey Data Release 7 (SDSS DR7) [75], and the Cosmic Microwave Background (CMB) radiation data from SevenYear Wilkinson Microwave Anisotropy Probe (WMAP) observations [76] to examine the teleparallel dark energy scenario. Since the model includes the scalar-field potential, we perform our analysis for three different potential cases, given by:

- Power-Law potential

This potential class is common in cosmology [77-81]. Although we can straightforwardly perform our analysis for an arbitrary exponent, for simplicity we focus on the most interesting quartic case

$$
V(\phi)=V_{0} \phi^{4} .
$$


- Exponential potential

Exponential potentials are also very common in the literature [82-85], which are necessary to be considered in every observational constraining analysis. In the following we use the ansatz

$$
V(\phi)=V_{0} e^{-\kappa \lambda \phi}
$$

- Inverse hyperbolic cosine potential

The use of hyperbolic cosine potential, or power-law functions of it, has also many cosmological implications [86-88]. Although we could perform our analysis for an arbitrary exponent, in the following we focus on the inverse case, namely:

$$
V(\phi)=\frac{V_{0}}{\cosh (\kappa \phi)} .
$$

We examine the constraints on the model parameters and the present values of the density parameters, following the $\chi^{2}$-method for the recent observational data. The detailed analysis method for SNIa, BAO and CMB data is summarized in Appendix. In general, we are interested in producing the likelihood contours for physically-interesting parameters, namely the present dark-energy equation-of-state parameter $w_{D E_{0}}$, the present matter density parameter $\Omega_{m 0}$ and the nonminimal coupling parameter $\xi$. We mention here that $\xi$ must always be bounded according to a physical constraint, namely it must lead to positive $\rho_{D E}$ and $H^{2}$ in relations (2.9) and (2.11), respectively. In practice, $\xi$ is found to be mainly negative (in our convention), and only a small window of positive values is theoretically allowed. In our analysis, for each of the three potentials, we fit three parameters, namely $w_{D E_{0}}$, the dimensionless Hubble parameter $h$ and $\Omega_{m 0}(\xi)$, and then we draw the likelihoodcontours for $1 \sigma$ and $2 \sigma$ confidence levels.

In Fig. 1 we present the likelihood contours for $w_{D E_{0}}$ and $\Omega_{m 0}$ with the teleparallel dark energy scenario under the quartic potential (3.1). As we observe, the scenario at hand is in agreement with observations, and as expected, it can describe both the quintessence and phantom regimes. Since the scalar field is canonical, it is a great advantage of the present model.

In Fig. 2 we present the likelihood contours for $w_{D E_{0}}$ and the nonminimal coupling parameter $\xi$, for the quartic potential (3.1). Interestingly enough we observe that the nonminimal coupling is favored by the data, and in particular a small $\xi$ is responsible for the quintessence regime, while a larger one leads to the phantom regime. Note that the best-fit values of $\left.w_{D E_{0}}\right|_{b . f} \approx-0.98$ is very close to the cosmological constant.

In Fig. 3 we present the likelihood contours for $w_{D E_{0}}$ and $\Omega_{m 0}$, for the teleparallel dark energy scenario under the exponential potential (3.2). As we observe, this scenario is consistent with observations, and it can describe both the quintessence and phantom regimes, with the phantom regime favored by the data. Furthermore, in Fig. 4 we present the likelihood contours for $w_{D E_{0}}$ and $\xi$, for the exponential potential (3.2). From this graph we deduce that a non-minimal coupling is favored by the data, and we observe that $w_{D E_{0}}{ }^{-}$ values close to the cosmological constant bound, either above or below it, can be induced 


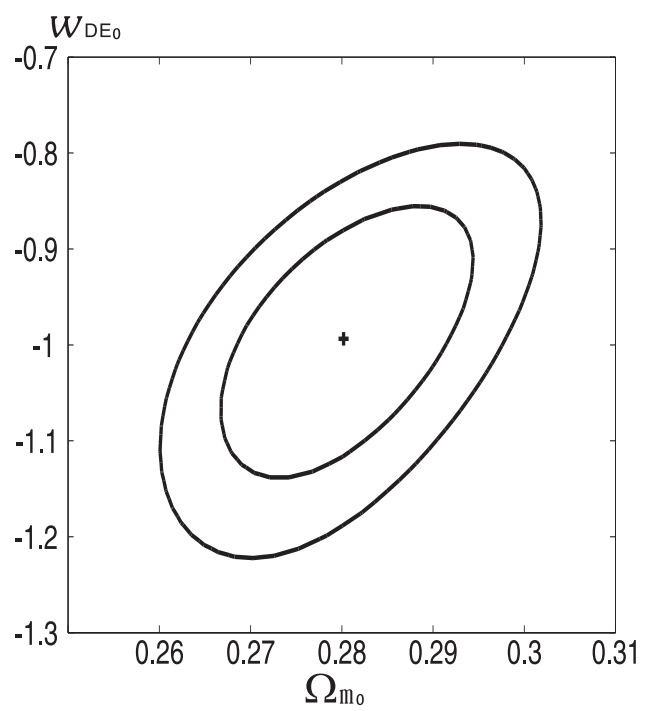

Figure 1. Contour plots of the present dark-energy equation-of-state parameter $w_{D E_{0}}$ versus the present matter density parameter $\Omega_{m 0}$ under SNIa, BAO and CMB observational data in the teleparallel dark energy scenario with the quartic potential $V(\phi)=V_{0} \phi^{4}$. The curves correspond to $1 \sigma$ and $2 \sigma$ confidence levels, respectively, and the cross marks the best-fit point.

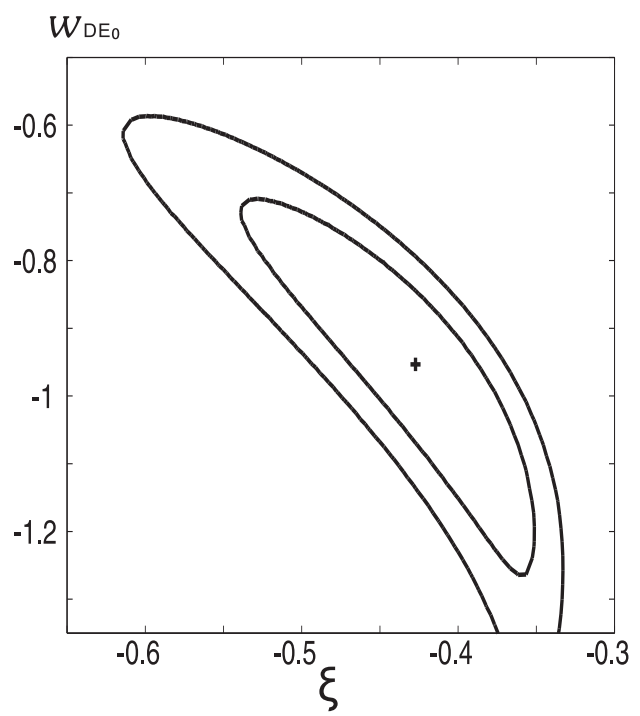

Figure 2. Contour plots of the present dark-energy equation-of-state parameter $w_{D E_{0}}$ versus the nonminimal coupling parameter $\xi$ under SNIa, BAO and CMB observational data, in the teleparallel dark energy scenario with the quartic potential $V(\phi)=V_{0} \phi^{4}$. The curves correspond to $1 \sigma$ and $2 \sigma$ confidence levels, respectively, and the cross marks the best-fit point.

by a relative large $\xi$-interval, which is an advantage of this scenario. It is interesting to mention that the exponential potential was used as an explicit example in [38], and our current analysis verifies its results. 


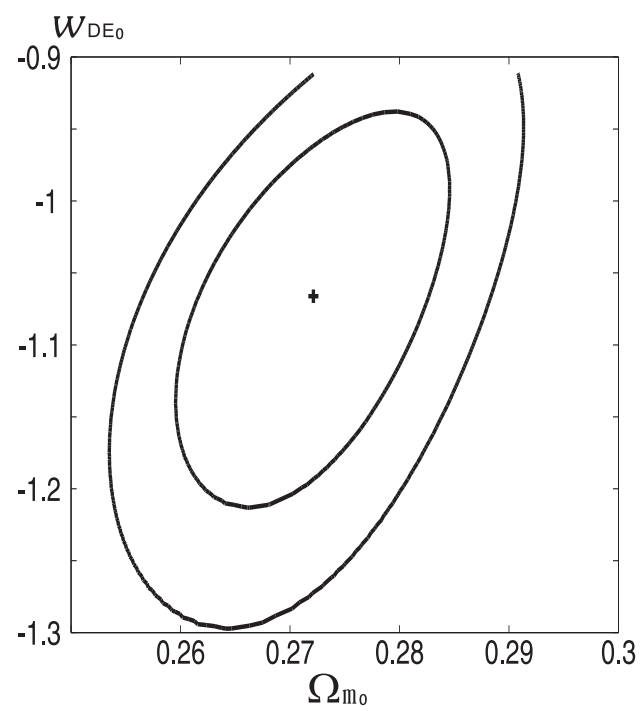

Figure 3. Legend is the same as Fig. 1 but with the exponential potential $V(\phi)=V_{0} e^{-\kappa \lambda \phi}$.

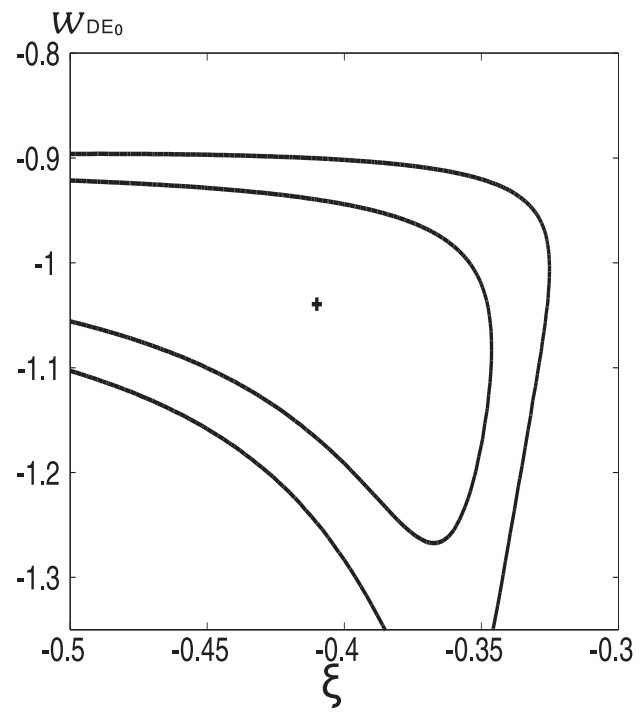

Figure 4. Legend is the same as Fig. 2 but with the exponential potential $V(\phi)=V_{0} e^{-\kappa \lambda \phi}$.

In Fig. 5 we depict the likelihood contours for $w_{D E_{0}}$ and $\Omega_{m 0}$, under the inverse hyperbolic cosine potential (3.3). As we can see, this scenario is consistent with observations. However, if we desire to avoid divergences in the $w_{D E}$ evolving history, we are restricted in the phantom regime. In addition, the best-fit value of $\left.w_{D E_{0}}\right|_{b . f} \approx-1.02$ is very close to the cosmological constant. Furthermore, in Fig. 6 we present the corresponding likelihood contours for $w_{D E_{0}}$ and $\xi$. Similarly to the previous cases we can see that the nonminimal coupling is favored by the data. It is interesting to note that $w_{D E_{0}}$-values close to the cosmological constant bound can be induced by a relative large $\xi$-interval. 


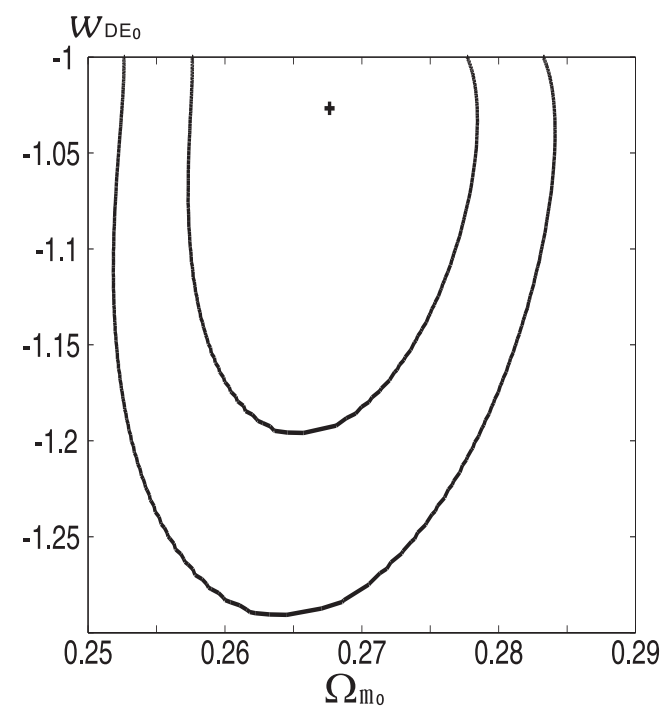

Figure 5. Legend is the same as Fig. 1 but with the inverse hyperbolic cosine potential $V(\phi)=$ $V_{0} / \cosh (\kappa \phi)$.

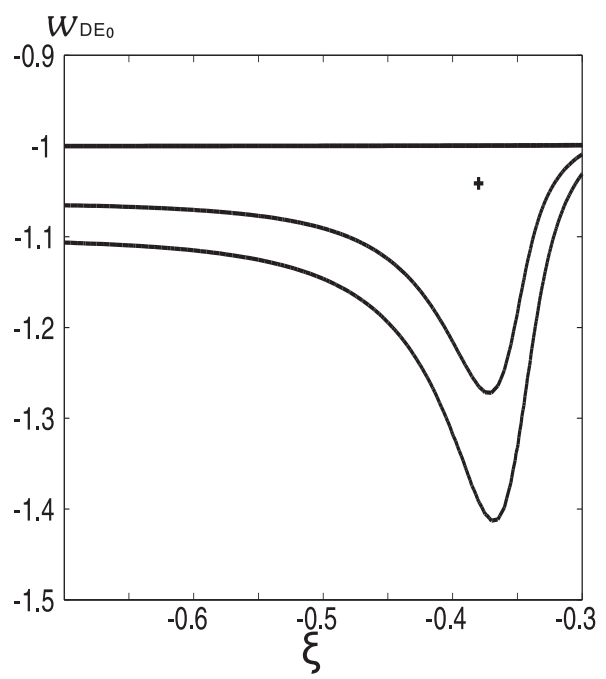

Figure 6. Legend is the same as Fig. 2 but with the inverse hyperbolic cosine potential $V(\phi)=$ $V_{0} / \cosh (\kappa \phi)$.

We close this section with a comment on the positive values of the nonminimal coupling $\xi$. As we mentioned above, the positivity requirement for $\rho_{D E}$ and $H^{2}$ leads $\xi$ to be negative, with only a small window of positive values theoretically allowed. Now, in practice, if we perform our fitting procedure in the positive $\xi$ region for the inverse hyperbolic cosine potential, we find that positive $\xi$ is excluded. However, for the quartic and exponential potentials we find the interesting result that for the theoretically allowed positive $\xi(0 \leq \xi \lesssim 0.2) w_{D E}$ is always very close to a constant $w_{D E_{0}}$ with $\left|w_{D E_{0}}-w_{D E}\right| \lesssim 10^{-3}$. 
The reason is that the scenario of the teleparallel dark energy for positive $\xi$ (sufficiently small in order for the positivity of $\rho_{D E}$ and $H^{2}$ not to be spoiled) always results in the stabilization of $w_{D E_{0}}$ close to the cosmological constant value, as can be proven by a detailed phase-space analysis [89]. Such a behavior is an advantage from both observational and theoretical point of view. We would like to note for comparison that, in the case of quintessence in the Einstein gravity and in the presence of a dust-like component (CDM and baryons), dark energy with an exactly constant $w_{D E}>-1$ is possible for the inverse hyperbolic sine potential in some power as was first independently shown in Refs. [90] and [91].

\section{Conclusions}

In the present work we have used observational data to impose constraints on the parameters of the teleparallel dark energy scenario [38], which is based on the teleparallel equivalent of General Relativity (TEGR), that is on its torsion instead of curvature formulation [39, 40]. In this model one adds a canonical scalar field, in which the dark energy sector is attributed, allowing also for a nonminimal coupling between the field and the torsion scalar. Thus, although the minimal case is completely equivalent with the standard quintessence, the nonminimal scenario has a richer structure, exhibiting the quintessencelike or phantom-like behavior, or experiencing the phantom-divide crossing [38].

In particular, we have fitted data from Type Ia Supernovae (SNIa), Baryon Acoustic Oscillations (BAO), and Cosmic Microwave Background (CMB) observations to constrain the present dark-energy equation-of-state parameter $w_{D E_{0}}$, the present matter density parameter $\Omega_{m 0}$ and the nonminimal coupling parameter $\xi$. Furthermore, in order to be general, for the scalar-field potential we have taken three ansatzes, namely the power-law, the exponential and the inverse hyperbolic cosine ones.

For the power-law (quartic) potential we have seen that teleparallel dark energy is compatible with observations and, as expected, it can describe both the quintessence and phantom regimes. Additionally, we have shown that the negative nonminimal coupling is favored by the data, and in particular a small $\xi$ is responsible for the quintessence regime, while a larger one leads to the phantom regime. For the exponential potential we have demonstrated that both the quintessence and phantom regimes can be described, with the phantom regime favored by the data. Moreover, we have found that a negative non-minimal coupling is favored and we have observed that $w_{D E_{0}}$-values close to the cosmological constant bound, either above or below it, can be induced by a relative large $\xi$-interval, which is an advantage of this scenario. For the inverse hyperbolic cosine potential we have shown that $w_{D E_{0}}$ is restricted in the phantom regime, while $\xi$ is restricted to negative values, with a relatively large $\xi$-interval being able to lead to $w_{D E_{0}}$-values close to -1 . We remark that positive values of $\xi$ are excluded for the inverse hyperbolic cosine potential, while for the power-law and exponential ones $w_{D E_{0}}$ is very close to -1 .

In summary, the scenario of the teleparallel dark energy is compatible with observations, for all the examined scalar-field potentials. Furthermore, although the scalar field is canonical, the model can describe both the quintessence and phantom regimes. These 
features are an advantage from both observational and theoretical point of view, and they make the scenario at hand a good candidate for the description of nature. Finally, the data favor a nonminimal coupling, and thus the model is distinguishable from the standard quintessence, since the two scenarios are equivalent only for the minimal coupling.

An interesting and necessary investigation would be to go beyond the background analysis, and examine observables that arise at the perturbation level, such are the growth of matter overdensities and the gravitational-wave spectrum, since these could also clarify possible Lorentz-violation problems that are not seen at the background level [92-94]. Since this study lies beyond the scope of the present manuscript, it is left for future investigation.

\section{Acknowledgments}

This work was partially supported by National Center of Theoretical Science and National Science Council (NSC-98-2112-M-007-008-MY3) of R.O.C. Two of us (CQG and CCL) would like to thank KITPC for the wonderful program of "Dark Matter and New Physics".

\section{A Analysis method for the observational data}

In this appendix, we explain the methods for the elaboration of observational data from Type Ia Supernovae (SNIa), Baryon Acoustic Oscillations (BAO) and Cosmic Microwave Background $(\mathrm{CMB})$ radiation. The $\chi^{2}$ of the combined observational data is given by

$$
\chi^{2}=\tilde{\chi}_{\mathrm{SN}}^{2}+\chi_{\mathrm{BAO}}^{2}+\chi_{\mathrm{CMB}}^{2}
$$

In our fitting procedure we use the simple $\chi^{2}$ method, rather than the Markov-chain Monte Carlo (MCMC) approach such as CosmoMC [95]. In the following we describe the calculation for the various $\chi_{i}^{2}$ of each observational dataset (for detailed explanations on the data analysis see e.g. [96, 97]).

\section{a. Type Ia Supernovae (SNe Ia)}

SNe Ia observations provide the information on the luminosity distance $D_{L}$ as a function of the redshift $z$. The theoretical distance modulus $\mu_{\mathrm{th}}$ is defined by

$$
\mu_{\mathrm{th}}\left(z_{i}\right) \equiv 5 \log _{10} D_{L}\left(z_{i}\right)+\mu_{0},
$$

where $\mu_{0} \equiv 42.38-5 \log _{10} h$, with $h \equiv H_{0} / 100 /\left[\mathrm{km} \mathrm{sec}^{-1} \mathrm{Mpc}^{-1}\right]$ [76]. The Hubble-free luminosity distance for the flat universe is described as

$$
D_{L}(z)=(1+z) \int_{0}^{z} \frac{d z^{\prime}}{E\left(z^{\prime}\right)}
$$

where $E(z) \equiv H(z) / H_{0}$, with

$$
\frac{H(z)}{H_{0}}=\sqrt{\Omega_{\mathrm{m}}^{(0)}(1+z)^{3}+\Omega_{\mathrm{r}}^{(0)}(1+z)^{4}+\Omega_{\mathrm{DE}}^{(0)}(1+z)^{3\left(1+w_{\mathrm{DE}}\right)}} .
$$


Here, $\Omega_{\mathrm{r}}^{(0)}=\Omega_{\gamma}^{(0)}\left(1+0.2271 N_{\text {eff }}\right)$, where $\Omega_{\gamma}^{(0)}$ is the present fractional photon energy density and $N_{\text {eff }}=3.04$ is the effective number of neutrino species [76]. We mention that $H(z)$ is evaluated by using numerical solutions of the Friedmann equation.

The $\chi^{2}$ of the SNe Ia data is given by

$$
\chi_{\mathrm{SN}}^{2}=\sum_{i} \frac{\left[\mu_{\mathrm{obs}}\left(z_{i}\right)-\mu_{\mathrm{th}}\left(z_{i}\right)\right]^{2}}{\sigma_{i}^{2}},
$$

where $\mu_{\mathrm{obs}}$ is the observed value of the distance modulus. In the following, subscriptions "th" and "obs" denote the theoretically predicted and observed values, respectively. $\chi_{\mathrm{SN}}^{2}$ is minimized with respect to $\mu_{0}$, which relates to the absolute magnitude, since the absolute magnitude of SNe Ia is not known. $\chi_{\mathrm{SN}}^{2}$ in (A.2) is expanded as [98]

$$
\chi_{S N}^{2}=A-2 \mu_{0} B+\mu_{0}^{2} C
$$

with

$$
\begin{aligned}
A & =\sum_{i} \frac{\left[\mu_{\mathrm{obs}}\left(z_{i}\right)-\mu_{\mathrm{th}}\left(z_{i} ; \mu_{0}=0\right)\right]^{2}}{\sigma_{i}^{2}}, \\
B & =\sum_{i} \frac{\mu_{\mathrm{obs}}\left(z_{i}\right)-\mu_{\mathrm{th}}\left(z_{i} ; \mu_{0}=0\right)}{\sigma_{i}^{2}}, \\
C & =\sum_{i} \frac{1}{\sigma_{i}^{2}} .
\end{aligned}
$$

Thus, the minimum of $\chi_{\mathrm{SN}}^{2}$ with respect to $\mu_{0}$ is expressed as

$$
\tilde{\chi}_{\mathrm{SN}}^{2}=A-\frac{B^{2}}{C} .
$$

In our analysis we apply expression (A.3) for the $\chi^{2}$ minimization and we use the Supernova Cosmology Project (SCP) Union2 compilation, which contains 557 supernovae [74], ranging from $z=0.015$ to $z=1.4$.

\section{b. Baryon Acoustic Oscillations (BAO)}

The distance ratio of $d_{z} \equiv r_{s}\left(z_{\mathrm{d}}\right) / D_{V}(z)$ is measured by BAO observations, where $D_{V}$ is the volume-averaged distance, $r_{s}$ is the comoving sound horizon and $z_{\mathrm{d}}$ is the redshift at the drag epoch [75]. The volume-averaged distance $D_{V}(z)$ is defined as [99]

$$
D_{V}(z) \equiv\left[(1+z)^{2} D_{A}^{2}(z) \frac{z}{H(z)}\right]^{1 / 3}
$$

where $D_{A}(z)$ is the proper angular diameter distance for the flat universe, defined by

$$
D_{A}(z) \equiv \frac{1}{1+z} \int_{0}^{z} \frac{d z^{\prime}}{H\left(z^{\prime}\right)}
$$


The comoving sound horizon $r_{s}(z)$ is given by

$$
r_{s}(z)=\frac{1}{\sqrt{3}} \int_{0}^{1 /(1+z)} \frac{d a}{a^{2} H\left(z^{\prime}=1 / a-1\right) \sqrt{1+\left(3 \Omega_{b}^{(0)} / 4 \Omega_{\gamma}^{(0)}\right) a}},
$$

where $\Omega_{b}^{(0)}=2.2765 \times 10^{-2} h^{-2}$ and $\Omega_{\gamma}^{(0)}=2.469 \times 10^{-5} h^{-2}$ are the current values of baryon and photon density parameters, respectively [76]. The fitting formula for $z_{\mathrm{d}}$ is given by [100]

$$
z_{\mathrm{d}}=\frac{1291\left(\Omega_{\mathrm{m}}^{(0)} h^{2}\right)^{0.251}}{1+0.659\left(\Omega_{\mathrm{m}}^{(0)} h^{2}\right)^{0.828}}\left[1+b_{1}\left(\Omega_{b}^{(0)} h^{2}\right)^{b 2}\right]
$$

with

$$
\begin{aligned}
& b_{1}=0.313\left(\Omega_{\mathrm{m}}^{0} h^{2}\right)^{-0.419}\left[1+0.607\left(\Omega_{\mathrm{m}}^{0} h^{2}\right)^{0.674}\right], \\
& b_{2}=0.238\left(\Omega_{\mathrm{m}}^{0} h^{2}\right)^{0.223} .
\end{aligned}
$$

The typical value of $z_{\mathrm{d}}$ is about 1021 for $\Omega_{\mathrm{m}}^{(0)}=0.276$ and $h=0.705$.

According to the BAO data from the Two-Degree Field Galaxy Redshift Survey (2dFGRS) and the Sloan Digital Sky Survey Data Release 7 (SDSS DR7) [75], the distance ratio $d_{z}$ at two redshifts $z=0.2$ and $z=0.35$ is measured to be $d_{z=0.2}^{\text {obs }}=0.1905 \pm 0.0061$ and $d_{z=0.35}^{\text {obs }}=0.1097 \pm 0.0036$, with the inverse covariance matrix:

$$
C_{\mathrm{BAO}}^{-1}=\left(\begin{array}{cc}
30124 & -17227 \\
-17227 & 86977
\end{array}\right) \text {. }
$$

Finally, the $\chi^{2}$ for the BAO data is calculated as

$$
\chi_{\mathrm{BAO}}^{2}=\left(x_{i, \mathrm{BAO}}^{\mathrm{th}}-x_{i, \mathrm{BAO}}^{\mathrm{obs}}\right)\left(C_{\mathrm{BAO}}^{-1}\right)_{i j}\left(x_{j, \mathrm{BAO}}^{\mathrm{th}}-x_{j, \mathrm{BAO}}^{\mathrm{obs}}\right),
$$

where $x_{i, \mathrm{BAO}} \equiv\left(d_{0.2}, d_{0.35}\right)$.

c. Cosmic Microwave Background (CMB) radiation

The CMB observational data are sensitive to the distance to the decoupling epoch $z_{*}[76]$. Hence, by using these data we obtain constraints on the model in the high redshift regime $(z \sim 1000)$.

The acoustic scale $l_{A}$ and the shift parameter $\mathcal{R}$ [101] are defined by

$$
\begin{aligned}
& l_{A}\left(z_{*}\right) \equiv\left(1+z_{*}\right) \frac{\pi D_{A}\left(z_{*}\right)}{r_{s}\left(z_{*}\right)}, \\
& \mathcal{R}\left(z_{*}\right) \equiv \sqrt{\Omega_{\mathrm{m}}^{(0)}} H_{0}\left(1+z_{*}\right) D_{A}\left(z_{*}\right),
\end{aligned}
$$

where $z_{*}$ is the redshift of the decoupling epoch, given by [102]

$$
z_{*}=1048\left[1+0.00124\left(\Omega_{b}^{(0)} h^{2}\right)^{-0.738}\right]\left[1+g_{1}\left(\Omega_{\mathrm{m}}^{(0)} h^{2}\right)^{g 2}\right],
$$


with

$$
g_{1}=\frac{0.0783\left(\Omega_{b}^{(0)} h^{2}\right)^{-0.238}}{1+39.5\left(\Omega_{b}^{(0)} h^{2}\right)^{0.763}}, \quad g_{2}=\frac{0.560}{1+21.1\left(\Omega_{b}^{(0)} h^{2}\right)^{1.81}} .
$$

We use the data from Seven-Year Wilkinson Microwave Anisotropy Probe (WMAP) observations [76] on CMB.

The $\chi^{2}$ of the CMB data is

$$
\chi_{\mathrm{CMB}}^{2}=\left(x_{i, \mathrm{CMB}}^{\mathrm{th}}-x_{i, \mathrm{CMB}}^{\mathrm{obs}}\right)\left(C_{\mathrm{CMB}}^{-1}\right)_{i j}\left(x_{j, \mathrm{CMB}}^{\mathrm{th}}-x_{j, \mathrm{CMB}}^{\mathrm{obs}}\right),
$$

where $x_{i, \mathrm{CMB}} \equiv\left(l_{A}\left(z_{*}\right), \mathcal{R}\left(z_{*}\right), z_{*}\right)$ and $C_{\mathrm{CMB}}^{-1}$ is the inverse covariance matrix. The data from WMAP7 observations [76] lead to $l_{A}\left(z_{*}\right)=302.09, \mathcal{R}\left(z_{*}\right)=1.725$ and $z_{*}=1091.3$ with the inverse covariance matrix:

$$
C_{\mathrm{CMB}}^{-1}=\left(\begin{array}{ccc}
2.305 & 29.698 & -1.333 \\
29.698 & 6825.27 & -113.180 \\
-1.333 & -113.180 & 3.414
\end{array}\right) .
$$

\section{References}

[1] A. G. Riess et al. [Supernova Search Team Collaboration], Observational evidence from supernovae for an accelerating universe and a cosmological constant, Astron. J. 116, 1009 (1998), [arXiv: astro-ph/9805201].

[2] S. Perlmutter et al. [Supernova Cosmology Project Collaboration], Measurements of Omega and Lambda from 42 high redshift supernovae, Astrophys. J. 517, 565 (1999), [arXiv: astro-ph/9812133].

[3] D. N. Spergel et al. [WMAP Collaboration], First year Wilkinson Microwave Anisotropy Probe (WMAP) observations: Determination of cosmological parameters, Astrophys. J. Suppl. 148, 175 (2003), [arXiv: astro-ph/0302209].

[4] M. Tegmark et al. [SDSS Collaboration], Cosmological parameters from SDSS and WMAP, Phys. Rev. D69, 103501 (2004), [arXiv: astro-ph/0310723].

[5] S. W. Allen, R. W. Schmidt, H. Ebeling, A. C. Fabian, L. van Speybroeck, Constraints on dark energy from Chandra observations of the largest relaxed galaxy clusters, Mon. Not. Roy. Astron. Soc. 353, 457 (2004), [arXiv: astro-ph/0405340].

[6] B. Ratra, P. J. E. Peebles, Cosmological Consequences of a Rolling Homogeneous Scalar Field, Phys. Rev. D37, 3406 (1988).

[7] C. Wetterich, Cosmology and the Fate of Dilatation Symmetry, Nucl. Phys. B302, 668 (1988).

[8] A. R. Liddle, R. J. Scherrer, A Classification of scalar field potentials with cosmological scaling solutions, Phys. Rev. D59, 023509 (1999), [arXiv:astro-ph/9809272]. [astro-ph/9809272].

[9] I. Zlatev, L. -M. Wang, P. J. Steinhardt, Quintessence, cosmic coincidence, and the cosmological constant, Phys. Rev. Lett. 82, 896 (1999), [arXiv:astro-ph/9807002].

[10] Z. -K. Guo, N. Ohta, Y. -Z. Zhang, Parametrization of quintessence and its potential, Phys. Rev. D72, 023504 (2005), [arXiv: astro-ph/0505253]. 
[11] S. Dutta, E. N. Saridakis, R. J. Scherrer, Dark energy from a quintessence (phantom) field rolling near potential minimum (maximum), Phys. Rev. D79, 103005 (2009), [arXiv:0903.3412].

[12] V. Sahni, S. Habib, Does inflationary particle production suggest Omega(m) less than 1?, Phys. Rev. Lett. 81, 1766 (1998), [arXiv:hep-ph/9808204].

[13] J. -P. Uzan, Cosmological scaling solutions of nonminimally coupled scalar fields, Phys. Rev. D59, 123510 (1999), [arXiv:gr-qc/9903004].

[14] N. Bartolo, M. Pietroni, Scalar tensor gravity and quintessence, Phys. Rev. D61, 023518 (2000), [arXiv:hep-ph/9908521].

[15] V. Faraoni, Inflation and quintessence with nonminimal coupling, Phys. Rev. D62, 023504 (2000), [arXiv:gr-qc/0002091].

[16] T. D. Saini, S. Raychaudhury, V. Sahni, A. A. Starobinsky, Reconstructing the cosmic equation of state from supernova distances, Phys. Rev. Lett. 85, 1162 (2000), [arXiv: astro-ph/9910231].

[17] B. Boisseau, G. Esposito-Farese, D. Polarski and A. A. Starobinsky, Reconstruction of a scalar tensor theory of gravity in an accelerating universe, Phys. Rev. Lett. 85, 2236 (2000) [arXiv:gr-qc/0001066].

[18] E. Elizalde, S. 'i. Nojiri, S. D. Odintsov, Late-time cosmology in (phantom) scalar-tensor theory: Dark energy and the cosmic speed-up, Phys. Rev. D70, 043539 (2004), [arXiv:hep-th/0405034].

[19] V. Faraoni, De Sitter attractors in generalized gravity, Phys. Rev. D70, 044037 (2004), [arXiv:gr-qc/0407021].

[20] R. Gannouji, D. Polarski, A. Ranquet and A. A. Starobinsky, Scalar-Tensor Models of Normal and Phantom Dark Energy, JCAP 0609, 016 (2006) [arXiv:astro-ph/0606287].

[21] M. Szydlowski, O. Hrycyna, A. Kurek, Coupling constant constraints in a nonminimally coupled phantom cosmology, Phys. Rev. D77, 027302 (2008), [arXiv:0710.0366].

[22] M. R. Setare, E. N. Saridakis, Non-minimally coupled canonical, phantom and quintom models of holographic dark energy, Phys. Lett. B671, 331 (2009), [arXiv:0810.0645].

[23] R. R. Caldwell, A Phantom menace?, Phys. Lett. B545, 23 (2002), [arXiv: astro-ph/9908168].

[24] R. R. Caldwell, M. Kamionkowski, N. N. Weinberg, Phantom energy and cosmic doomsday, Phys. Rev. Lett. 91, 071301 (2003), [arXiv:astro-ph/0302506].

[25] S. 'i. Nojiri, S. D. Odintsov, Quantum de Sitter cosmology and phantom matter, Phys. Lett. B562, 147 (2003), [arXiv: hep-th/0303117]. [hep-th/0303117].

[26] V. K. Onemli, R. P. Woodard, Quantum effects can render $w$ i-1 on cosmological scales, Phys. Rev. D70, 107301 (2004), [arXiv:gr-qc/0406098]. [gr-qc/0406098].

[27] S. Dutta and R. J. Scherrer, Dark Energy from a Phantom Field Near a Local Potential Minimum, Phys. Lett. B 676, 12 (2009), [arXiv:0902.1004].

[28] C. Kaeonikhom, B. Gumjudpai, E. N. Saridakis, Observational constraints on phantom power-law cosmology, Phys. Lett. B695, 45 (2011), [arXiv:1008.2182].

[29] B. Feng, X. -L. Wang, X. -M. Zhang, Dark energy constraints from the cosmic age and 
supernova, Phys. Lett. B607, 35 (2005), [arXiv:astro-ph/0404224].

[30] Z. -K. Guo, Y. -S. Piao, X. -M. Zhang, Y. -Z. Zhang, Cosmological evolution of a quintom model of dark energy, Phys. Lett. B608, 177 (2005), [arXiv: astro-ph/0410654].

[31] B. Feng, M. Li, Y. -S. Piao, X. Zhang, Oscillating quintom and the recurrent universe, Phys. Lett. B634, 101 (2006), [arXiv : astro-ph/0407432]. [astro-ph/0407432].

[32] W. Zhao and Y. Zhang, Quintom models with an equation of state crossing -1, Phys. Rev. D73, 123509 (2006), [arXiv: astro-ph/0604460].

[33] M. R. Setare, E. N. Saridakis, Quintom model with $O(N)$ symmetry, JCAP 0809, 026 (2008), [arXiv:0809.0114].

[34] L. P. Chimento, M. I. Forte, R. Lazkoz, M. G. Richarte, Internal space structure generalization of the quintom cosmological scenario, Phys. Rev. D79, 043502 (2009), [arXiv:0811.3643].

[35] Y. -F. Cai, E. N. Saridakis, M. R. Setare, J. -Q. Xia, Quintom Cosmology: Theoretical implications and observations, Phys. Rept. 493, 1 (2010), [arXiv: :0909.2776].

[36] E. J. Copeland, M. Sami and S. Tsujikawa, Dynamics of dark energy, Int. J. Mod. Phys. D 15, 1753 (2006), [arXiv: hep-th/0603057].

[37] S. 'i. Nojiri, S. D. Odintsov, Introduction to modified gravity and gravitational alternative for dark energy, [arXiv:hep-th/0601213].

[38] C. Q. Geng, C. C. Lee, E. N. Saridakis, Y. P. Wu, 'Teleparallel' Dark Energy, Phys. Lett. B704, 384 (2011), [arXiv:1109.1092].

[39] A. Unzicker and T. Case, Translation of Einstein's attempt of a unified field theory with teleparallelism, [arXiv: physics/0503046].

[40] K. Hayashi and T. Shirafuji, New General Relativity, Phys. Rev. D 19, 3524 (1979), [Addendum-ibid. D 24, 3312 (1982)].

[41] S. H. Chen, J. B. Dent, S. Dutta and E. N. Saridakis, Cosmological perturbations in $f(T)$ gravity, Phys. Rev. D 83, 023508 (2011), [arXiv: 1008.1250].

[42] J. B. Dent, S. Dutta, E. N. Saridakis, $f(T)$ gravity mimicking dynamical dark energy. Background and perturbation analysis, JCAP 1101, 009 (2011), [arXiv:1010.2215].

[43] Weitzenböck R., Invarianten Theorie, Nordhoff, Groningen, The Netherlands (1923).

[44] J. W. Maluf, Hamiltonian formulation of the teleparallel description of general relativity, J. Math. Phys. 35 (1994) 335.

[45] H. I. Arcos and J. G. Pereira, Torsion Gravity: a Reappraisal, Int. J. Mod. Phys. D 13, 2193 (2004), [arXiv:gr-qc/0501017].

[46] G. R. Bengochea and R. Ferraro, Dark torsion as the cosmic speed-up, Phys. Rev. D 79, 124019 (2009), [arXiv:0812.1205].

[47] E. V. Linder, Einstein's Other Gravity and the Acceleration of the Universe, Phys. Rev. D 81, 127301 (2010), [arXiv:1005.3039].

[48] R. Myrzakulov, Accelerating universe from $F(T)$ gravities, [arXiv:1006.1120].

[49] P. Wu, H. W. Yu, $f(T)$ models with phantom divide line crossing, Eur. Phys. J. C71, 1552 (2011), [arXiv: 1008.3669]. 
[50] K. Bamba, C. Q. Geng, C. C. Lee, Comment on 'Einstein's Other Gravity and the Acceleration of the Universe, [arXiv:1008.4036].

[51] R. Zheng, Q. G. Huang, Growth factor in $f(T)$ gravity, JCAP 1103, 002 (2011), [arXiv: 1010.3512].

[52] K. Bamba, C. Q. Geng, C. C. Lee, L. W. Luo, Equation of state for dark energy in $f(T)$ gravity, JCAP 1101, 021 (2011), [arXiv:1011.0508].

[53] T. Wang, Static Solutions with Spherical Symmetry in $f(T)$ Theories, Phys. Rev. D84, 024042 (2011), [arXiv: 1102.4410].

[54] K. K. Yerzhanov, S. R. Myrzakul, I. I. Kulnazarov and R. Myrzakulov, Accelerating cosmology in $F(T)$ gravity with scalar field, [arXiv: 1006.3879].

[55] R. J. Yang, Conformal transformation in $f(T)$ theories, Europhys. Lett. 93, 60001 (2011), [arXiv: 1010.1376].

[56] P. Wu, H. W. Yu, Observational constraints on $f(T)$ theory, Phys. Lett. B693, 415 (2010), [arXiv: 1006 . 0674].

[57] G. R. Bengochea, Observational information for $f(T)$ theories and Dark Torsion, Phys. Lett. B695, 405 (2011), [arXiv:1008.3188].

[58] P. Wu, H. W. Yu, The dynamical behavior of $f(T)$ theory, Phys. Lett. B692, 176 (2010), [arXiv: 1007.2348].

[59] Y. Zhang, H. Li, Y. Gong, Z. -H. Zhu, Notes on $f(T)$ Theories, JCAP 1107, 015 (2011), [arXiv:1103.0719].

[60] R. Ferraro, F. Fiorini, Non trivial frames for $f(T)$ theories of gravity and beyond, Phys. Lett. B702, 75 (2011), [arXiv: 1103.0824].

[61] Y. F. Cai, S. H. Chen, J. B. Dent, S. Dutta, E. N. Saridakis, Matter Bounce Cosmology with the $f(T)$ Gravity, [arXiv:1104.4349]. [arXiv:1104.4349 [astro-ph.CO]].

[62] S. Chattopadhyay, U. Debnath, Emergent universe in chameleon, $f(R)$ and $f(T)$ gravity theories, Int. J. Mod. Phys. D20, 1135 (2011), [arXiv:1105.1091].

[63] M. Sharif, S. Rani, $F(T)$ Models within Bianchi Type I Universe, Mod. Phys. Lett. A26, 1657 (2011), [arXiv: 1105.6228].

[64] H. Wei, X. P. Ma, H. Y. Qi, $f(T)$ Theories and Varying Fine Structure Constant Phys. Lett. B703, 74 (2011), [arXiv: 1106.0102].

[65] R. Ferraro, F. Fiorini, Cosmological frames for theories with absolute parallelism, [arXiv: 1106.6349].

[66] R. X. Miao, M. Li, Y. G. Miao, Violation of the first law of black hole thermodynamics in $f(T)$ gravity, [arXiv:1107.0515].

[67] C. G. Boehmer, A. Mussa, N. Tamanini, Existence of relativistic stars in $f(T)$ gravity, [arXiv:1107.4455].

[68] H. Wei, H. Y. Qi, X. P. Ma, Constraining $f(T)$ Theories with the Varying Gravitational Constant, [arXiv:1108.0859].

[69] S. Capozziello, V. F. Cardone, H. Farajollahi, A. Ravanpak, Cosmography in f(T)-gravity, [arXiv:1108.2789].

[70] P. Wu, H. Yu, The stability of the Einstein static state in $f(T)$ gravity, Phys. Lett. B703, 
223 (2011), [arXiv: 1108.5908].

[71] M. H. Daouda, M. E. Rodrigues, M. J. S. Houndjo, Static Anisotropic Solutions in $f(T)$ Theory, [arXiv:1109.0528].

[72] K. Bamba, C. Q. Geng, Thermodynamics of cosmological horizons in $f(T)$ gravity, [arXiv:1109.1694].

[73] H. Wei, Dynamics of Teleparallel Dark Energy, [arXiv:1109.6107].

[74] R. Amanullah et al., Spectra and Light Curves of Six Type Ia Supernovae at $0.511<z<$ 1.12 and the Union2 Compilation, Astrophys. J. 716, 712 (2010), [arXiv:1004.1711].

[75] W. J. Percival et al., Baryon Acoustic Oscillations in the Sloan Digital Sky Survey Data Release 7 Galaxy Sample, Mon. Not. Roy. Astron. Soc. 401, 2148 (2010), [arXiv:0907.1660].

[76] E. Komatsu et al., Seven-Year Wilkinson Microwave Anisotropy Probe (WMAP) Observations: Cosmological Interpretation, [arXiv:1001.4538].

[77] A. de la Macorra, C. Stephan-Otto, Quintessence restrictions on negative power and condensate potentials, Phys. Rev. D65, 083520 (2002), [arXiv:astro-ph/0110460].

[78] J. P. Kneller, L. E. Strigari, Inverse power law quintessence with non-tracking initial conditions, Phys. Rev. D68, 083517 (2003), [arXiv:astro-ph/0302167].

[79] X. Zhang, Coupled quintessence in a power-law case and the cosmic coincidence problem, Mod. Phys. Lett. A20, 2575 (2005), [arXiv:astro-ph/0503072].

[80] E. N. Saridakis, Phantom evolution in power-law potentials, Nucl. Phys. B819, 116 (2009), [arXiv: 0902 . 3978].

[81] E. N. Saridakis, Quintom evolution in power-law potentials, Nucl. Phys. B830, 374 (2010), [arXiv: 0903.3840].

[82] P. G. Ferreira, M. Joyce, Structure formation with a selftuning scalar field, Phys. Rev. Lett. 79, 4740 (1997), [arXiv: astro-ph/9707286].

[83] E. J. Copeland, A. RLiddle, D. Wands, Exponential potentials and cosmological scaling solutions, Phys. Rev. D57, 4686 (1998), [arXiv:gr-qc/9711068].

[84] E. J. Copeland, M. Sami, S. Tsujikawa, Dynamics of dark energy, Int. J. Mod. Phys. D15, 1753 (2006), [arXiv:hep-th/0603057]. [hep-th/0603057].

[85] X. -m. Chen, Y. -g. Gong, E. N. Saridakis, Phase-space analysis of interacting phantom cosmology, JCAP 0904, 001 (2009), [arXiv:0812.1117].

[86] V. Sahni, L. M. Wang, A New cosmological model of quintessence and dark matter, Phys. Rev. D62, 103517 (2000), [arXiv:astro-ph/9910097].

[87] V. Sahni, M. Sami, T. Souradeep, Relic gravity waves from brane world inflation, Phys. Rev. D65, 023518 (2002), [arXiv:gr-qc/0105121].

[88] P. Singh, M. Sami, N. Dadhich, Cosmological dynamics of phantom field, Phys. Rev. D68, 023522 (2003), [arXiv:hep-th/0305110].

[89] C. Xu, E. N. Saridakis, Y. g. Gong, In preparation.

[90] V. Sahni and A. A. Starobinsky, The Case for a positive cosmological Lambda term, Int. J. Mod. Phys. D 9, 373 (2000) [arXiv:astro-ph/9904398].

[91] L. A. Urena-Lopez and T. Matos, A New cosmological tracker solution for quintessence, 
Phys. Rev. D 62, 081302 (2000) [arXiv:astro-ph/0003364].

[92] T. P. Sotiriou, B. Li, J. D. Barrow, Generalizations of teleparallel gravity and local Lorentz symmetry, Phys. Rev. D83, 104030 (2011), [arXiv: 1012.4039].

[93] B. Li, T. P. Sotiriou, J. D. Barrow, Large-scale Structure in $f(T)$ Gravity, Phys. Rev. D83, 104017 (2011), [arXiv:1103.2786].

[94] M. Li, R. X. Miao, Y. G. Miao, Degrees of freedom of $f(T)$ gravity, JHEP 1107, 108 (2011), [arXiv: 1105.5934].

[95] A. Lewis and S. Bridle, Cosmological parameters from CMB and other data: a Monte-Carlo approach, Phys. Rev. D 66, 103511 (2002), [arXiv:astro-ph/0205436].

[96] L. Yang, C. C. Lee, L. W. Luo, C. Q. Geng, Observational Constraints on Exponential Gravity, Phys. Rev. D82, 103515 (2010), [arXiv: 1010.2058].

[97] M. Li, X. D. Li and X. Zhang, Comparison of dark energy models: A perspective from the latest observational data, Sci. China Phys. Mech. Astron. 53, 1631 (2010), [arXiv: 0912.3988].

[98] L. Perivolaropoulos, Constraints on linear-negative potentials in quintessence and phantom models from recent supernova data, Phys. Rev. D 71, 063503 (2005),

[arXiv: astro-ph/0412308].

[99] D. J. Eisenstein et al. [ SDSS Collaboration ], Detection of the baryon acoustic peak in the large-scale correlation function of SDSS luminous red galaxies, Astrophys. J. 633, 560 (2005), [arXiv: astro-ph/0501171].

[100] D. J. Eisenstein and W. Hu, Baryonic Features in the Matter Transfer Function, Astrophys. J. 496, 605 (1998), [arXiv:astro-ph/9709112].

[101] J. R. Bond, G. Efstathiou and M. Tegmark, Forecasting Cosmic Parameter Errors from Microwave Background Anisotropy Experiments, Mon. Not. Roy. Astron. Soc. 291, L33 (1997), [arXiv: astro-ph/9702100].

[102] W. Hu and N. Sugiyama, Small scale cosmological perturbations: An Analytic approach, Astrophys. J. 471, 542 (1996), [arXiv:astro-ph/9510117]. 